\section{Dr. Claudepierre and Dr. Thomas reply}

\section{To the Editor:}

We thank Prof. Toussirot for providing data on new-onset psoriasis in patients taking rituximab (RTX) for rheumatoid arthritis (RA) ${ }^{1}$. His case report is well documented, with a dermatologist confirmation and a typical illustration of psoriasis, although no biopsy was performed. In this case, a link between RTX therapy and new-onset psoriasis is suggested. Nevertheless, it may also be hypothesized that emergence of psoriasis is independent of RTX treatment and is eventually triggered by the decrease of corticosteroids; indeed, data on progression of psoriasis lesion after the second course of RTX are not available, and the causality of RTX in the occurrence of psoriasis is thus at most "possible."

In our large observational prospective cohort, among 1927 patients taking RTX for RA, only 2 experienced new-onset psoriasis, including the patient in whom the diagnosis of psoriasis was established retrospectively from patient's description; further, these 2 cases had only a "doubtful" causality ${ }^{2}$. Our study did not support a role for RTX therapy in new-onset psoriasis, but because of a weak incidence rate with a wide CI, we could not definitely eliminate it. We also pointed out that, in contrast to tumor necrosis factor- $\alpha$ antagonists, the case reports for RTX were not linked by a consistent clinical pattern, time-course, or site involvement ${ }^{3,4,5,6}$. For example, the 2 new-onset psoriasis cases described in our study cleared after a few weeks, while in the case described by Toussirot, psoriasis lesions remained stable.

Thus, no firm conclusions can be drawn, and if RTX could induce psoriasis lesions, the incidence is very rare, regarding its prescription. Nevertheless, clinicians should be aware of a possible but uncertain role of B cell depletion with RTX in the development of psoriasis, in a few patients.
PASCAL CLAUDEPIERRE, MD, Department of Rheumatology; LAURE THOMAS, MD, Department of Pharmacovigilance, Hopital Henri-Mondor, 51 Boulevard de Lattre de Tassigny, 94000 Créteil, France. Address correspondence to Prof. Claudepierre;

E-mail: pascal.claudepierre@hmn.aphp.fr

\section{REFERENCES}

1. Toussirot E. New onset of psoriasis in a patient with rheumatoid arthritis treated with rituximab [letter]. J Rheumatol 2013; 40:1230-1.

2. Thomas L, Canoui-Poitrine F, Gottenberg JE, Economu-Dubosc A, Medkour F, Chevalier X, et al. Incidence of new-onset and flare of preexisting psoriasis during rituximab therapy for rheumatoid arthritis: data from the French AIR registry [review]. J Rheumatol 2012;39:893-8.

3. Dass S, Vital EM, Emery P. Development of psoriasis after B cell depletion with rituximab. Arthritis Rheum 2007;56:2715-8.

4. Mielke F, Schneider-Obermeyer J, Dörner T. Onset of psoriasis with psoriatic arthropathy during rituximab treatment of non-Hodgkin lymphoma. Ann Rheum Dis 2008;67:1056-7.

5. Markatseli TE, Kaltsonoudis ES, Voulgari PV, Zioga A, Drosos AA. Induction of psoriasis skin lesions in a patient with rheumatoid arthritis treated with rituximab. Clin Exp Rheumatol 2009;27:1002-4.

6. Hardcastle SA, Gibbs S, Williamson L. Atypical psoriasis following rituximab for rheumatoid arthritis. J Rheumatol 2012;39:1303-4.

J Rheumatol 2013;40:7; doi:10.3899/jrheum.130258 